\title{
La enseñanza del inglés en las escuelas multigrado: equidad o inequidad
}

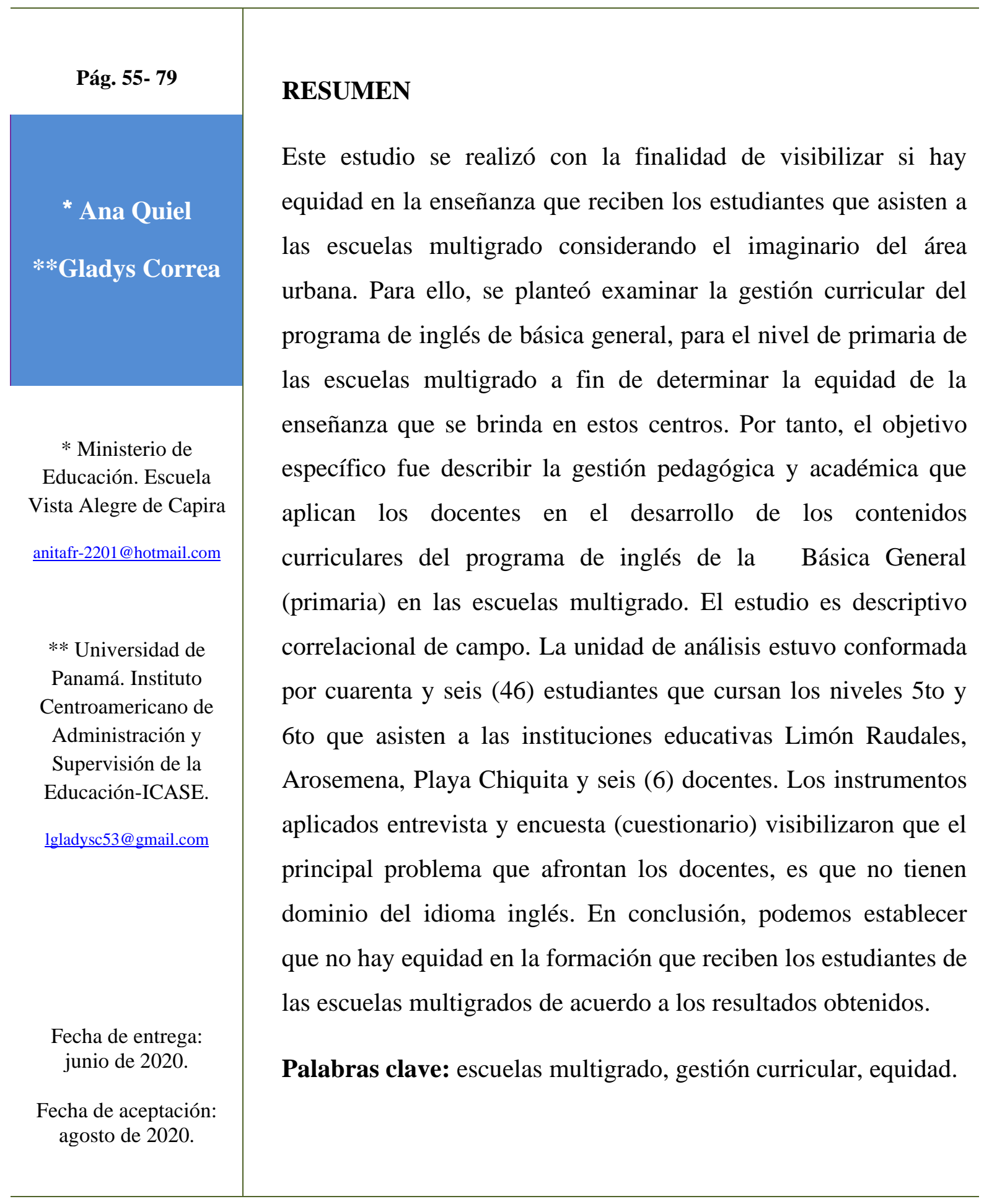




\title{
The teaching of the english language at multigrade schools: equity or inequity.
}

\begin{abstract}
This study was carried out in order to make visible if there is equity in the teaching received by students who attend multigrade schools, located in urban areas. It was proposed to examine the curricular management of the general basic English program for the primary levels of multigrade schools. The aim of this is to determine the equity of the education provided. Therefore, the specific objective was to describe the pedagogical and academic management applied by teachers in the development of the curricular contents of the General Basic (primary) English program in multigrade schools. The study is a descriptive correlational field. The unit of analysis was made up of forty-six (46) students attending the $5^{\text {th }}$ and $6^{\text {th }}$ at the Limon Raudales, Arosemena, Playa Chiquita schools plus six teachers. The interview and survey (questionnaire) made visible that the main problem faced by the teachers was not being able to handle the English language. In conclusion, there is no equity in the formation received by the multigrade school students according to the obtained results.
\end{abstract}

Keywords: Multigrade schools, curriculum management, equity. 


\section{INTRODUCCIÓN}

En el campo educativo, como en el resto de las ciencias, la investigación se ha constituido en una actividad precisa y elemental, a tal punto que busca tratar los problemas relativos a la cotidianidad para encontrar posibles soluciones con la finalidad de mejorar la educación, como es el caso de la indagación sobre el currículo, los métodos de enseñanza $\mathrm{y}$ factores inherentes al acto educativo tales como: tiempo de aprendizaje, medios y materiales, organización, clima de la clase, y procesos de interacción o comunicación.

Partiendo de esta premisa, la base de una formación académica depende en gran parte de su educación básica, ya que en ella es donde se conciben los cimientos de futuros profesionales, por ende, es importante que se pueda lograr en el educando un desarrollo eficaz durante sus primeros pasos de formación.

Sin embargo, bajo esta realidad existen necesidades diversas, que obstaculizan este principio para lograr una educación de calidad. Este es el caso de las escuelas rurales, especialmente las escuelas multigrado en las cuales los maestros, directivos, padres de familia y el alumnado, se enfrentan a problemas como: un currículum atomizado y no ajustado para el tipo de escuela, infraestructura inadecuada, carencia de mobiliario con las características mínimas y falta de servicios de salud, por mencionar algunos; aunado a esto, la inasistencia de estudiantes, maestros con poco dominio en la temática, especialmente en asignaturas como inglés, considerando, además, que un porcentaje elevado de los maestros héroes que aceptan laborar en estas áreas solo cuentan con el título base o un bachillerato que les permite ejercer la profesión.

Así mismo, el poco apoyo por parte de la administración y la falta de una supervisión más profunda son características muy acentuadas en los países emergentes como el nuestro. Al respecto, Carmena y Regidor, (2014) nos señalan que las escuelas rurales hacen referencia al conjunto de formas de escolarización específicas de unas áreas geográficas denominadas zonas rurales, cuyas características son: el bajo ratio alumno/profesor, los agrupamientos por ciclos o etapas, la desvinculación con el medio rural de los maestros, 
el predominio de los centros estatales, la coexistencia de centros completos e incompletos, la alta inmovilidad del profesorado y la deficiencia o inexistencia de instalaciones.

Visto de esta forma, la educación en los medios rurales es una realidad que se convierte en una oportunidad que se brinda a cientos de niños y jóvenes que no cuentan con recursos o capacidad para asistir a colegios en las grandes ciudades. Por ende, es prioritario que la educación sea igual en estas zonas, pues el hecho de estar escolarizado es un punto a favor de esa identidad social.

Ahora bien, con la realidad descrita, se hace pertinente y necesario la existencia de aulas multigrado, en la cual un maestro o maestra enseña a dos o más grados al mismo tiempo, esto constituye la realidad educativa predominante de la escuela primaria de áreas rurales en la región.

En Panamá existen cerca de 24 mil centros educativos de primaria, de los cuales el 73\% son multigrados, donde el $32,1 \%$ de la matrícula total de primaria es atendida en estas escuelas y el 33,9\% de maestros de primaria enseña en ellas. Ministerio de Educación (2018). Así mismo, se puede señalar que alrededor del $90 \%$ de los centros educativos de primaria del medio rural son atendidos por docentes con más de un grado a su cargo.

En este orden de ideas, es de resaltar que la escuela rural es eminentemente multigrado, a semejanza de lo que ocurre en otros países de la región y de otras partes del mundo, donde las escuelas multigrados se ubican en las zonas más apartadas del territorio y atienden, principalmente, a las poblaciones campesinas, indígenas y pobres.

No obstante, lo anterior, la escuela multigrado a pesar de encontrarse en tal situación, debe buscar pedagógicamente la forma y manera de adecuar el currículo a las necesidades del alumnado. Por ende, la política educativa, la cual tiene como referente el modelo monogrado de la escuela primaria debe adecuar programas de formación de maestros, permitiendo tomar en cuenta esta realidad educativa para así ofrecer de forma adecuada el pensum que busque la máxima enseñanza en las diversas asignaturas. 
Por consiguiente, la asignatura de inglés al ser una materia establecida por el Estado y el Ministerio de Educación de Panamá mediante la LEY No. 2 de 14 de enero de 2003, como indispensable para la formación del alumnado de primaria, debe ser enseñada en las escuela multigrado, sin olvidar la problemática que enfrentan los docentes que laboran en estos centros donde deben enfrentar no solo enseñar el inglés sino también el castellano considerando que la mayoría de estos centros se ubican en áreas indígenas que además tienen su propio idioma. Por ello, se hace relevante tener la preparación, la disposición y el uso de métodos pedagógicos, que les permita atender los programas de estudio establecidos por el ente rector del sistema.

Con la intención de tener un mapeo más amplio de este tipo de escuelas que vienen a resolver una necesidad en las áreas de difícil acceso, para atender la cobertura, en muchas ocasiones, en detrimento de la calidad, se puede señalar que uno de los problemas es el currículo que se ofrece, el cual fue pensado para escuelas completas, lo que significa que los maestros que laboran en los centros multigrado deben realizar adecuaciones. Otro aspecto a señalar, es que muchos de los maestros que se desempeñan en estas áreas ostentan un título cuya formación de base es un bachiller en ciencias, letras, tecnológico o cualquiera de los 18 bachilleratos que ofrece el sistema educativo panameño y desconocen las estrategias para atender varios grados simultáneamente, y por otro lado, deben asumir el rol directivo cuyas tareas absorben una mayor cantidad de tiempo en detrimento de las acciones pedagógicas que al sumar los días de reuniones y actividades de índole administrativas llevan a la suspensión de clases. Sin embargo, no podemos ubicar en esta situación al bachillerato pedagógico, el cual tiene como propósito la formación en estrategias metodológicas que luego, perfeccionan en el nivel superior. Esta formación es recibida en la Normal Superior Juan Demóstenes Arosemena.

Toda esta panorámica del contexto nos abrió un sinnúmero de puertas para investigar, pero en estos momentos, nos enfocamos al estudio de la enseñanza del inglés en las escuelas multigrado. Tal como se planteó, el diseño curricular de la educación básica, nivel primario, está pensado para escuelas completas, no así para multigrado. Por tanto, el desarrollo del inglés en este tipo de escuelas presenta grandes dificultades. Si analizamos 
todo ese proceso de formación de los maestros que obtienen título universitario, el inglés que reciben durante dos semestres no se desdobla en lo que el programa de la básica presenta dentro de su diseño curricular. Por tanto, desconocen los temas, las estrategias y actividades para ser enseñado en los diferentes grados que corresponden a la básica general de las escuelas multigrado. Si bien es cierto existe la Ley que estipula el inglés como una asignatura de carácter obligatoria a la que se le asigna una cantidad de horas; al adentrarnos en el funcionamiento de las escuelas multigrado, se ha podido constatar que no se pensó en cómo se van a cubrir estas horas, puesto que la distribución del horario y de atención en este tipo de escuela es diferenciado, por tanto, hay que organizar y planificar de manera distinta a las escuelas cuya organización es completa.

Además, otro de los problemas que enfrentan los docentes de las escuelas multigrado es la poca visita de los supervisores al área, recibiendo insuficiente o nula orientación que les permita implementar estrategias novedosas no solo para que los estudiantes logren esos conocimientos, sino para retenerlos en los centros educativos. Pero dentro de esa gama de problemas también miramos la infraestructura de estos centros educativos las cuales no son óptimas, ya que siendo escuelas ranchos en su gran mayoría hay carencia de energía eléctrica, lo que imposibilita el acceso a la tecnología siendo un obstáculo mayor si se piensa que a través de ella, se podría desarrollar con mayores posibilidades la enseñanza del inglés, considerando que existen numerosos programas que se pueden utilizar. En cuanto al mobiliario, tampoco es el más adecuado, comparativamente es de menor calidad y no guarda ni siquiera las mismas condiciones del existente en las escuelas urbanas.

Por lo expuesto, el estudio busca determinar la gestión curricular para el desarrollo del programa de inglés del nivel de básica general primaria en escuelas multigrado, de la zona 9 de la Dirección Regional de Educación de Panamá Oeste.

El mismo parte de las inquietudes y dificultades que manifiestan los docentes de escuelas multigrado, dado que, al momento de la planificación y aplicación de los programas de la asignatura de inglés, se evidencia que no existen adecuaciones en consideración a las particularidades de este tipo de escuelas. Así por ejemplo, la carga horaria de las asignaturas fundamentales en los planes de estudio de la básica general utilizados en 
multigrado, establecen una cantidad de horas semanales, que muchas veces reducen el tiempo para la enseñanza del inglés. A partir de lo anterior se formulan los siguientes problemas de investigación:

- ¿Cómo es la gestión curricular del programa de inglés de básica general de las escuelas multigrados del nivel primario?

- ¿Cuál será la efectividad de la gestión curricular del programa de inglés de la básica general (primaria) en las escuelas multigrado para ofrecer las mismas condiciones pedagógicas a los estudiantes de estas escuelas?

- ¿Cuáles serán los elementos obstaculizadores de la gestión pedagógica para el desarrollo del programa de inglés de la básica general (primaria) que permiten una equidad en la enseñanza de las escuelas multigrado?

Como objetivo general se consideró, "Determinar la gestión curricular del programa de inglés de básica general para el nivel primaria de las escuelas multigrado de la zona 9 de la Regional de Panamá Oeste; con la finalidad de especificar la equidad de la enseñanza”.

Consecuente con esto, se proponen los siguientes objetivos específicos:

- Describir la gestión pedagógica y académica que aplican los docentes para el desarrollo de los contenidos curriculares del programa de inglés de la básica general en las escuelas multigrado del nivel primario, con la finalidad de lograr los mismos niveles de las escuelas del área urbana.

- Detallar la efectividad de la gestión curricular del programa de inglés de la básica general en las escuelas multigrado del nivel primario con la finalidad de evidenciar equidad de la información desarrollada en estas escuelas.

- Identificar los elementos obstaculizadores de la gestión pedagógica para el desarrollo del programa de inglés de la básica general (primaria) que impiden la equidad de la enseñanza y del aprendizaje. 
Como se ha podido evidenciar la educación en el área rural se imparte en escuelas multigrado. Allí predominan condiciones físicas estructurales que limitan el desempeño de los maestros. Estas escuelas cuentan con pocos docentes, los cuales deben enfrentar condiciones laborales difíciles por la falta de insumos, así mismo, las condiciones de aislamiento se expresan en muy poca asesoría pedagógica, supervisión y escasa capacitación, lo que se expresa en algunos indicadores educativos como: deserción y promoción.

Ante los problemas señalados se planteó la hipótesis: El proceso de gestión curricular para la enseñanza del inglés en las escuelas multigrado, nivel de primaria, permite la equidad de la enseñanza, similar al de las escuelas urbanas.

En relación a la equidad, el Plan Estratégico de Educación - Políticas Educativas a la Acción, 2019-2024 del Ministerio de Educación de Panamá, presenta el Eje N.2 de Equidad Educativa, para el cual propone un conjunto de programas dirigidos a asegurar este aspecto, tal es el caso de: Acceso a la educación en todas las culturas y regiones comarcales, Atención de la población con necesidades educativas especiales. Remediación y reinserción donde las actividades, y programas priorizados se enfocan en : Ampliar la cobertura en áreas rurales y comarcas indígenas, Programa Estudiar sin Hambre, Fortalecimiento de la Educación Intercultural Bilingüe, Modelo y estrategia de atención efectiva de la población con discapacidad, Ampliar cobertura y niveles educativos de las poblaciones con necesidades especiales a través de las mejoras en prácticas pedagógicas, Diseño del marco legal y estrategias educativas de remediación, Diseño y oferta de remediación para los niveles de Educación Básica General y Media, Programa Encontrando el Camino Correcto (p.7).

En esa misma línea de pensamiento este documento establece la Misión, Visión, Valores y Principios de la gestión y establece, dentro de los valores la Equidad, la cual define como la acción para “Asegurar que todas las niñas y niños completen su educación, de manera especial aquellos que se encuentren en condición de pobreza, exclusión y vulnerabilidad" (p.8). 
Jiménez (2016), en su libro La escuela rural, analiza la situación de los maestros y alumnos advirtiendo una doble exclusión, propia del subdesarrollo rural y el atraso socioeconómico, la ausencia de reconocimiento de su rico y potencial territorio y sus desaprovechados recursos en las decisiones educativas, políticas y sociales.

Se puede deducir entonces que el enfoque de equidad reconoce las diferencias individuales y socioeconómicas desfavorables para poder corregirlas. Por ello, para que exista equidad en la educación se requiere que coexista igualdad de oportunidades y capacidades para eliminar los obstáculos sociales que impiden la libre competencia entre los individuos, pero también que dicha competencia sea justa y que los resultados de esa libertad se traduzcan en capacidades que generen ventajas para los desfavorecidos. Es así que, existe una acepción clara de justicia en el término de equidad, pero también de inclusión para los grupos sociales. (Bracho et ál., 2009).

Por otro lado, Emma (2016), manifiesta que la enseñanza del inglés analiza el aprendizaje de nuevo vocabulario en lengua inglesa relacionado con el campo de la informática. Plantea la hipótesis de que, como consecuencia de la ubicación del centro rural, el nivel social y de vida de los alumnos y de la organización del centro, los alumnos se encuentran en desventaja a la hora de aprender vocabulario informático en inglés.

El aprendizaje de una segunda lengua suele presentar dificultades y crear frustraciones en los alumnos, por lo que el maestro debe procurar en todo momento que los materiales utilizados para el mismo fin sean motivadores.

Por otro lado, Alcalá (2013), describe las debilidades y fortalezas que representa la enseñanza de este idioma en las áreas rurales, se trata de un estudio descriptivo, donde aplica entrevistas y encuestas a los maestros, padres de familia y estudiantes, para conocer la situación actual del inglés; considera importante que todos los niños escolarizados, tengan los mismos derechos de aprendizaje, incluyendo entre ellos el inglés, debido al gran auge mundial que tiene esta lengua y la gran importancia que tiene en la actualidad, por el componente social y cultural. El autor concluye y pondera la importancia de que 
los estudiantes al momento de culminar sus estudios primarios y emigrar a ciudades más grandes para continuar sus estudios, tengan el nivel académico igual al de las escuelas urbanas y que esto no sea un impedimento ni motivo para que exista diferencia alguna debido a su procedencia.

Con el fin de comprender que son escuelas multigrado Casarini (2010), nos plantea que son aquellas donde los maestros atienden a dos o más grupos a la vez, y de éstas existen tres tipos: tridocentes, bidocentes y unitarias; por lo cual su organización difiere en cada una de ellas, es decir, en las escuelas tridocentes hay tres maestros, uno para cada ciclo. Los del primer ciclo atienden a los de $1^{\circ}$ y $2^{\circ}$ grado; los del segundo a $3^{\circ}$ y $4^{\circ}$ y $\operatorname{los}$ del tercero a $5^{\circ}$ y $6^{\circ}$.

En consecuencia, también tenemos instituciones bidocentes que pueden organizarse de dos formas, la primera de ellas es que un maestro atiende a los tres primeros grados y el otro a los tres últimos, o un maestro trabaja con el primer ciclo y otro con los grados de $3^{\circ}$ a $6^{\circ}$. El último tipo de escuelas multigrado, es la unitaria, y como su nombre lo indica, un solo docente atiende a todos los grados.

Para esto estaban capacitados los maestros normalistas, cuya profesión debe ser restablecida; considerando que la escuela multigrado tiene una lógica y una organización distinta a la de la escuela graduada, y requiere por ende un tratamiento también distinto en todos los ámbitos: administración, currículo, pedagogía, organización y manejo del tiempo, infraestructura, evaluación, etc. Contar con vivienda para el docente es asimismo fundamental si la escuela se asienta en lugares apartados.

En Panamá, la escuela unitaria o multigrado todavía sigue siendo un reto para el director quien trabaja directamente con todos los alumnos. Algunos maestros se quejan de la falta de libros para todos los alumnos, por lo que es casi imposible dar clase de manera "simultánea".

La población se compone básicamente por niños de comunidades rurales y niños indígenas con un alto nivel de reprobación, deserción y bajos resultados educativos. Las 
condiciones sociales de dicha población, pobreza, exclusión, desigualdad e inequidad, impiden una educación eficaz y de calidad en las aulas. Esto no solo quiere decir que estas poblaciones no pueden acceder a la educación, sino que tampoco se puede desarrollar toda la información que corresponde a los diferentes grados ya que no se tiene en cuenta el entorno en el que se ejerce este derecho. Sin embargo, a pesar de las limitantes la enseñanza del idioma inglés en el Estado Panameño desde la enseñanza primaria hasta la universitaria constituye uno de los objetivos de primordial importancia, la cual ha estado orientada a formar ciudadanos con una cultura general integral y con un pensamiento humanista, científico y creador.

Según Guillen (2016), se ha implementado en muchas escuelas la enseñanza de una lengua extranjera a una edad temprana, como resultado de esta situación, estudios de adquisición del lenguaje apuntan a que cuando los niños aprenden segundas lenguas a una edad temprana, desarrollan sus sistemas gramaticales de manera no consciente y natural.

Este aprendizaje puede estar relacionado a la entrada de la lengua extranjera. Algunas de las ideas prácticas y básicas que plantea el referido autor para la orientación y apoyo de los docentes de lenguas extranjeras en la escuela multigrado son las siguientes:

-Aprendizaje Temprano, las Rutinas, las Transiciones.

Como apunta Cameron (2001), las señas no verbales (fotos, carteles, letreros) son especialmente importantes en este contexto de aprendizaje porque anticipan explícitamente a los aprendices la actividad que se va a llevar a cabo.

Así mismo, se brinda posibilidades de adaptar un entorno específico a uno en el que la adquisición del idioma sea más sencilla por medio de carteles o medios audiovisuales que estimulen el interés por la lengua extranjera.

Partiendo de esta premisa, de acuerdo con Bonhomme, et ál. (2015), en cuanto a la práctica del docente, señala que: "Los docentes pueden realizar diversas prácticas durante sus clases, sus preferencias por una u otras pueden dar origen a patrones identificables 
con ciertos tipos de docencia, los que se pueden ordenar con una tipología por el referido estudio" (p.385).

El objetivo fue categorizar los patrones o perfiles de docencia que caracterizan a los profesores participantes en:

- Tradicional, práctica asociada a la trasmisión directa del contenido y al constante monitoreo del mismo, el docente expone, cuestiona, y los estudiantes normalmente usan guías que son evaluadas;

- Práctica orientada al estudiante, describe un clima de apoyo y sintonía con el estudiante e instrucción individualizada, en donde los estudiantes trabajan en grupos pequeños, desarrollan opinión y trabajan según sus capacidades individuales; y

- Práctica alternativa, implica la participación activa del estudiante en la producción del conocimiento, ya sea por medio de debates, proyectos de investigación o elaboración de ensayos.

Bajo estos indicativos, el docente hoy tiene ante sí grandes retos: El primero de ellos es que la sociedad se ha convertido en una gran distribuidora de conocimientos y esto cambia profundamente el rol del docente en particular en aquel que desempeña funciones en las escuelas multigrado.

Sin duda, la sociedad hoy en día enmarca el todo, por ende, el docente es quien se ocupa de poner el conocimiento a disposición de las nuevas generaciones. No obstante, a diferencia de lo que ocurría en tiempos anteriores, ahora la sociedad está sometida a vertiginosos cambios que plantean continuamente nuevas problemáticas, exigiendo a las personas, múltiples competencias procedimentales (iniciativa, creatividad, uso de herramientas, estrategias de resolución de problemas, trabajo en equipo, entre otros) para crear y disponer el conocimiento preciso al estudiantado, el cual les permita afrontar los retos y metas que conlleven al éxito. 
Como insumo importante para este estudio se listan los objetivos generales del programa de inglés para la educación básica general del nivel primario del Ministerio de Educación de Panamá, los cuales se encuentran en los programas curriculares de la educación básica general del MEDUCA:

- Lograr que el idioma inglés sea un instrumento para contribuir al desarrollo individual y colectivo. Permitiéndoles servir a nuestra sociedad y nación.

- Adquirir destrezas lingüísticas básicas que permitan comunicarse en forma oral.

- Adquirir vocabulario básico y común en inglés.

- Desarrollar habilidades y destrezas que permitan utilizar el idioma inglés en forma creativa.

- Mostrar interés en el proceso de aprender y comprender el uso de un idioma extranjero con métodos innovadores.

- Utilizar el idioma extranjero para comunicarse con el (la) docente y otros estudiantes.

- Aumentar las destrezas semánticas.

- Mostrar valores morales, culturales, sociales y familiares.

- La expresión oral y escrita, en forma correcta.

- Adquirir conocimientos lingüísticos básicos del idioma inglés que les permita la comunicación fluida, efectiva y comprensiva dentro del entorno en que se desenvuelven encaminándose por el mundo de la competitividad para incorporarse al mundo laboral.

A continuación, se enuncian los objetivos por áreas de enseñanza en la asignatura de inglés. 
Tabla 1

Objetivos por áreas de enseñanza de la asignatura de inglés

\begin{tabular}{cc}
\hline $\begin{array}{c}\text { Áreas de } \\
\text { enseñanza }\end{array}$ & Objetivos \\
\hline Área 1 & Family, School, Community, Country \\
\hline Área 2 & People, (sentiments, body parts). \\
\hline Área 3 & Health, Nutrition, Food \\
\hline Área 4 & Ecology (Plants ,animals) \\
\hline Área 5 & Recreation (Tourism,sports,holidays) \\
\hline Área 6 & Weather, climate, Time numbers, Monetary units \\
\hline Área 7 & Transportation, Communication \\
\hline Área 8 & Literature \\
\hline Área 9 & Technology, Energy \\
\hline
\end{tabular}

Fuente: Tomado del Programa Oficial del Ministerio de Educación 2018.

\section{METODOLOGÍA}

El estudio está destinado a ofrecer un análisis, el cual se ajusta a una investigación cuantitativa, de tipo campo, nivel descriptivo correlacional. La unidad de análisis estuvo conformada por cuarenta y seis (46) estudiante que cursan los niveles 5to y 6to que asisten a las instituciones educativas; Limón Raudales, Arosemena y Playa Chiquita y seis (6) profesionales docentes que laboran en las mencionadas instituciones. La muestra utilizada es de tipo intencional selectivo, debido que se empleó un sustraendo de la población para ser objeto de estudio.

Para la obtención de la información se utilizó la encuesta y la entrevista estructurada.

Así mismo, se aplicó una prueba piloto para realizar el proceso de validación, obteniéndose resultados, a través del coeficiente de Alpha de Cron Bach, (tanto para la encuesta del docente, como para el alumnado) de 0,90; siendo de esta forma un instrumento óptimo.

La documentación que sirvió de apoyo desde la teoría son: libros cuyo enfoque desarrolla la temática de la enseñanza del inglés en las área rurales, consulta a la Ley $\mathrm{N}^{\circ} 2$ de 14 de 
enero de 2003, que determina la obligatoriedad de la enseñanza del inglés en todos los centros educativos del país con carácter de obligatoriedad, estadísticas del Ministerio de Educación y estadísticas que presenta la Contraloría General de la República de Panamá del último censo realizado oficialmente (año 2010), entre otros.

\section{RESULTADOS}

La unidad de análisis se ubicó en la zona $N^{\circ} 9$ de la Región de Panamá Oeste, donde se seleccionaron tres centros educativos de zonas rurales, que son atendidos por seis docentes, todos egresados de la Facultad de Educación de la Universidad de Panamá, que tienen como base bachilleratos en letras y comercio, pero ninguno con bachillerato pedagógico. Los seis docentes cuentan con licenciatura en educación primaria y dos poseen estudios de postgrado.

En la entrevista realizada a los maestros, se les consultó sobre las mayores dificultades que se han presentado en la gestión curricular del programa de inglés, entre sus respuestas se destacan las siguientes:

- No tiene dominio del inglés.

- El programa tiene muchos objetivos.

- El factor tiempo no les alcanza para desarrollar esta asignatura cuando se le debe dar mayor énfasis a las fundamentales por ser centros multigrado.

- No se les ha enseñado como se realiza una planificación para esta asignatura.

- No reciben capacitaciones o seminarios para poder enseñar este idioma. 
$\mathrm{Al}$ respecto, la Tabla 2 presenta las principales opiniones de los docentes, según escuela.

\section{Tabla 2}

¿Cuáles han sido las mayores dificultades que se le han presentado en la gestión curricular del programa de estudio de esta asignatura?

Escuela Mayores dificultades identificadas por los maestros

Limón Raudales K1.-La mayor dificultad que se presenta es la pronunciación ya que no domina mucho el inglés.

K1.- Para los docentes no hay una capacitación o seminario en lo curricular y no hay libros para poder trabajar.

Arosemena

K2.- Una de las grandes dificultades es el tiempo por el hecho de atender varios grados a la vez que no son especialistas en el ramo.

K1.- El tiempo, además, no son docentes de inglés, por lo tanto, nunca cubren todos los objetivos de inglés.

Playa Chiquita

K2.- Una de las mayores dificultades en la gestión curricular es que el programa cuenta con muchos objetivos está muy recargado.

K3.- Su mayor dificultad es la planificación.

Fuente: Entrevista realizada a los docentes de las escuelas que conforman unidad de análisis 2019.

$\mathrm{K}=$ identifica respuestas de cada docente

Con relación a los años de ejercer la docencia en este nivel, en escuelas multigrado, podemos señalar, que:

- $\quad$ un docente tiene de 1-3 años,

- cuatro docentes tienen de 4-6 años,

- $\quad$ y un docente tiene más de 7 años en escuelas multigrado. 
Con respecto a la categoría de estas escuelas, de acuerdo a la normativa existente que regula la clasificación según la cantidad de docentes que laboran en ésta, se evidencia que:

- Unidocente: 1 escuela (Limón Raudales). Aquí el maestro atiende los 6 grados.

- Polidocente: Dos escuelas (Arosemena y Playa Chuiquita). Hay más de un maestro, aunque atienden cada uno a más de 1 grado.

Al tener la distribución de las nueve áreas por grado y la cantidad de contenidos y objetivos de inglés por cada área se puede detectar que para el primer grado se deben desarrollar 8 áreas, 15 contenidos y 23 objetivos, en el caso del tercer grado se desarrollan 8 áreas 17 contenidos y 25 objetivos y para el quinto grado son 9 áreas 13 contenidos y 26 objetivos. Ver Tabla 3.

La pregunta que surge es cómo desarrollar toda esta información en las escuelas multigrado cuando ellas deben cumplir con un horario que está establecido, y por otro lado, que si estas escuelas estuviesen en zonas indígenas se tendría que cumplir con lo que estableció la Ley 88 del 22 de noviembre del 2010, que reconoce la obligatoriedad de educar a la población indígena en sus propios idiomas y contextos culturales, así como la organización institucional, la definición del contenido del currículo, la capacitación docente, el acceso a fondos para su desarrollo, los vínculos con el crédito educativo y la participación de las autoridades indígenas en esta política educativa.

Situación que tiende a aumentar, si se tiene en cuenta que el reconocimiento jurídico de las lenguas y alfabetos indígenas promueve cambios institucionales, entre ellos, la creación de las direcciones regionales de Educación para las comarcas Emberá Wounaan y Ngäbe Buglé. Se hace mayor énfasis en la lectura y escritura de las lenguas indígenas, además en la participación de las autoridades indígenas durante la formulación de esta política en sus territorios. 


\section{Tabla 3}

Áreas por grado y distribución de contenidos y objetivos, según grado.

\begin{tabular}{|c|c|c|c|c|c|c|c|c|c|c|c|c|c|c|c|c|c|}
\hline \multirow[t]{3}{*}{ Áreas } & \multirow[t]{3}{*}{ Áreas por grado } & \multicolumn{4}{|c|}{ Total } & \multirow{2}{*}{\multicolumn{2}{|c|}{$1^{\circ}$}} & \multirow{2}{*}{\multicolumn{2}{|c|}{$2^{\circ}$}} & \multirow{2}{*}{\multicolumn{2}{|c|}{$3^{\circ}$}} & \multirow{2}{*}{\multicolumn{2}{|c|}{$4^{\circ}$}} & \multirow{2}{*}{\multicolumn{2}{|c|}{$5^{\circ}$}} & \multirow{2}{*}{\multicolumn{2}{|c|}{$6^{\circ}$}} \\
\hline & & \multicolumn{2}{|c|}{ Contenidos } & \multicolumn{2}{|c|}{ Objetivos } & & & & & & & & & & & & \\
\hline & & Número & Porcentaje & Número & Porcentaje & Cont. & Obj. & Cont. & Obj. & Cont. & Obj. & Cont. & Obj. & Cont. & Obj. & Cont. & Obj. \\
\hline AREA 1 & Family ,SchooL, Community, Country & 16 & 19.0 & 20 & 14.2 & 3 & 4 & 5 & 4 & 3 & 4 & 2 & 4 & 2 & 2 & 1 & 2 \\
\hline AREA 2 & People, (sentiments, body parts). & 11 & 13.1 & 20 & 14.2 & 4 & 4 & 2 & 5 & 2 & 4 & 1 & 3 & 1 & 2 & 1 & 2 \\
\hline AREA 3 & Health, Nutrition, Food & 6 & 7.1 & 16 & 11.3 & 1 & 2 & 1 & 2 & 1 & 4 & 1 & 3 & 1 & 3 & 1 & 2 \\
\hline AREA 4 & Ecology ( Plants , animals) & 9 & 10.7 & 20 & 14.2 & 2 & 5 & 2 & 5 & 2 & 4 & 1 & 2 & 1 & 2 & 1 & 2 \\
\hline AREA 5 & Recreation (Tourism,sports,holidays) & 9 & 10.7 & 16 & 11.3 & 1 & 2 & 1 & 1 & 3 & 3 & 1 & 3 & 1 & 3 & 2 & 4 \\
\hline AREA 6 & $\begin{array}{l}\text { Weather, climate, Time numbers, Monetary } \\
\text { units }\end{array}$ & 15 & 17.9 & 17 & 12.1 & 2 & 2 & 1 & 1 & 4 & 3 & 4 & 3 & 3 & 6 & 1 & 2 \\
\hline AREA 7 & Transportation, Communication & 8 & 9.5 & 14 & 9.9 & 1 & 2 & 1 & 1 & 1 & 2 & 2 & 2 & 2 & 3 & 1 & 4 \\
\hline AREA 8 & Literature & 7 & 8.3 & 14 & 9.9 & 1 & 2 & 1 & 2 & 1 & 1 & 1 & 1 & 1 & 3 & 2 & 5 \\
\hline AREA 9 & Technology, Energy & 3 & 3.6 & 4 & 2.8 & 0 & 0 & 0 & 0 & 0 & 0 & 2 & 2 & 1 & 2 & 0 & 0 \\
\hline & TOTALES & 84 & 100.0 & 141 & 100.0 & 15 & 23 & 14 & 21 & 17 & 25 & 15 & 23 & 13 & 26 & 10 & 23 \\
\hline
\end{tabular}


Al indagar como es la atención de los docentes por grado en las escuelas que sirvieron como unidad de análisis, se pudo establecer como se distribuye la atención de los alumnos en los tres centros educativos.

\section{Tabla 4}

Escuelas y Grados atendidos por Docente en la escuela multigrado

\begin{tabular}{|c|c|c|c|c|c|c|c|}
\hline \multirow{3}{*}{ Escuela } & \multicolumn{6}{|c|}{ Grados atendidos por docente } & \multirow{3}{*}{ Total } \\
\hline & \multirow{2}{*}{$\begin{array}{c}\begin{array}{c}\text { Todos los } \\
\text { grados }\end{array} \\
\text { De 1ro a 6to }\end{array}$} & \multicolumn{2}{|c|}{ Tres grados } & \multicolumn{3}{|c|}{ Dos grados } & \\
\hline & & $\begin{array}{c}\text { 1ero, } 2 \text { do } \\
\text { y 6to }\end{array}$ & $\begin{array}{c}\text { 3ero, } \\
\text { 4to y } \\
5 \text { to }\end{array}$ & $\begin{array}{l}\text { 1ero y } \\
2 \mathrm{do}\end{array}$ & $\begin{array}{l}\text { 3er y } \\
4 \text { to }\end{array}$ & $\begin{array}{l}\text { 5to y } \\
6 \text { to }\end{array}$ & \\
\hline Limón Raudales & 1 & & & & & & 1 \\
\hline Arosemena & & 1 & 1 & & & & 2 \\
\hline Playa Chiquita & & & & 1 & 1 & 1 & 3 \\
\hline Total de docentes & 1 & 2 & & & 3 & & \\
\hline
\end{tabular}

Fuente: Encuesta aplicada en el año 2019.

Como observamos en la Tabla 4, la escuela Limón Raudales, un solo maestro tiene bajo su responsabilidad los 6 grados en un horario que va de 8:00 am - 1:30 pm. En el caso de las escuelas polidocentes, Arosemena y Playa Chiquita, encontramos que: 2 docentes atienden 3 grados cada uno, y 3 docentes atienden 2 grados cada uno, respectivamente y en el mismo horario. La atención proporcionada por los docentes, de acuerdo al horario y la cantidad de grados que atienden lleva a pensar que si no existen guías y una planificación adecuada los niños y jóvenes que asisten a estos centros, no cubren los esenciales mínimos para poder asistir con un porcentaje de seguridad al séptimo grado de la básica general.

Con el objetivo de ubicarnos directamente en la enseñanza del inglés se preguntó a los docentes el dominio que tienen del idioma y se detectó que cinco docentes manifestaron que tiene un nivel básico del idioma y un solo docente un nivel medio. 
En ese orden de ideas se preguntó si recibían algún tipo de capacitación en la didáctica para la enseñanza del inglés y cuál era la frecuencia en que la reciben y las respuestas obtenidas evidencian que un $50 \%$ de los maestros ocasionalmente han recibido estas capacitaciones y el otro $50 \%$ señaló que nunca han recibido capacitación en la didáctica para la enseñanza del inglés.

Sin embargo, los maestros, aunque no tengan el dominio realizan su mejor esfuerzo para cumplir con el desarrollo de la asignatura.

Es importante, señalar también, a este respecto que la LEY No. 2 (14 de enero de 2003) Publicada en la Gaceta Oficial No. 24720 de 16 de enero de 2003 indica que esta asignatura debe ser desarrollada de acuerdo al grado que atiende y a la disponibilidad de la carga horaria de la escuela multigrado y que no existe una normativa que establezca cuantas horas por asignaturas se deben dar en este tipo de escuelas. De allí, que solamente se establecen los períodos de descanso y queda a criterio del maestro, organizar el horario de atención tratando de cubrir las 4 asignaturas fundamentales con la cantidad de horas establecidas en el plan de estudio de la educación básica nivel primario.

No obstante, es importante resaltar, que en esta cantidad de horas tampoco se cumplen a cabalidad por la atención que le dedica el docente a los diferentes grupos que tiene bajo su responsabilidad. Aspecto este que tiene mayor visibilidad cuando se le pregunta por el cumplimiento de la carga horaria. En la Tabla 5 se puede apreciar el dominio del idioma inglés que tienen los docentes por escuela multigrado para el desarrollo de la enseñanza del inglés. 


\section{Tabla 5}

Dominio que tienen los docentes del idioma inglés

\section{Docentes por escuelas}

\begin{tabular}{lcccccc}
\multirow{2}{*}{ Nivel } & \multicolumn{2}{c}{ Limón Raudales } & \multicolumn{2}{c}{ Arosemena } & \multicolumn{2}{c}{ Playa Chiquita } \\
\cline { 2 - 7 } & $\mathrm{N}^{\mathbf{o}}$ & $\%$ & $\mathrm{~N}^{\mathbf{0}}$ & $\%$ & $\mathrm{~N}^{\mathbf{2}}$ & $\%$ \\
\hline Totales & 1 & $17 \%$ & 2 & $33 \%$ & 3 & $50 \%$ \\
\hline Básico & 1 & $17 \%$ & 2 & $33 \%$ & 2 & $33 \%$ \\
Medio & 0 & $0 \%$ & 0 & $0 \%$ & 1 & $17 \%$ \\
Avanzado & 0 & $0 \%$ & 0 & $0 \%$ & 0 & $0 \%$ \\
\hline
\end{tabular}

Fuente: Encuesta Elaborada en el año 2019.

De lo anterior se destaca que, cinco (5) docentes reconocen poseer un nivel básico para la enseñanza del inglés, estos están ubicados, uno en la escuela Limón Raudales, dos en Arosemena y dos de Playa Chiquita. Por otro lado, un docente manifestó que su nivel de dominio del inglés es medio (Playa Chiquita), por lo que se puede inferir que tienen un conocimiento básico.

Respecto a la acción pedagógica de los docentes para la enseñanza del inglés, los docentes respondieron lo siguiente. Ver tabla 6.

\section{Tabla 6}

Acción pedagógica del docente en relación al desarrollo de la enseñanza del inglés

Datos proporcionados por los docentes

$\begin{array}{lll}\text { Nivel } & \text { Limón Raudales } & \text { Arosemena Chiquita }\end{array}$

\begin{tabular}{lcccccc} 
& $\mathrm{N}^{\mathbf{o}}$ & $\%$ & $\mathrm{~N}^{\mathrm{o}}$ & & $\mathrm{N}^{\mathrm{o}}$ & $\%$ \\
\hline Totales & 1 & $17 \%$ & 2 & $33.0 \%$ & 3 & $50 \%$ \\
\hline Bajo & 0 & $0 \%$ & 1 & $16.6 \%$ & 1 & $16.6 \%$ \\
Medio & 1 & $16.6 \%$ & 1 & $16.6 \%$ & 2 & $33 \%$ \\
Alto & 0 & $0 \%$ & 0 & $0 \%$ & 0 & $0 \%$ \\
\hline
\end{tabular}

Fuente: Encuesta Elaborada en el año 2019. 
En general, un 66\% de los docentes manifestaron que en el desarrollo de la enseñanza del inglés su acción pedagógica tiene un nivel medio, mientras que un 33\% señala que su nivel es bajo en relación a la acción pedagógica para la enseñanza del inglés.

De igual forma se consultó a los estudiantes sobre la cantidad de veces que se da inglés en la semana y en la Tabla 7 se muestran las respuestas.

\section{Tabla 7}

Cantidad de veces que se da inglés en la semana, según estudiantes

Datos proporcionados por los estudiantes

\begin{tabular}{ccccccc} 
Veces semanales & \multicolumn{2}{c}{ Limón Raudales } & \multicolumn{2}{c}{ Arosemena } & \multicolumn{2}{c}{ Playa Chiquita } \\
\cline { 2 - 7 } & $\mathrm{N}^{\mathbf{o}}$ & $\%$ & $\mathrm{~N}^{\mathbf{o}}$ & $\%$ & $\mathrm{~N}^{\mathbf{0}}$ & $\%$ \\
\hline Totales & 8 & 17 & 20 & 44 & 18 & 39 \\
\hline Una 1 vez & 0 & $0 \%$ & 12 & 27 & 14 & 30 \\
Más de una 1 vez & 8 & 17 & 8 & 17 & 4 & 9 \\
\hline
\end{tabular}

Fuente: Encuesta Elaborada en el año 2019.

Con referencia a cuantas veces a la semana se imparte el inglés, los estudiantes manifestaron en un $57 \%$, que una vez a la semana, mientras que un $43 \%$ señala que más de una vez. Sin embargo, es muy significativo que en los datos proporcionados por los estudiantes se señaló que en todas las escuelas se imparte el inglés más de una vez dentro del horario de clases establecido, aunque esto no significa que se cubra con el horario reglamentado por el Ministerio de Educación, recordando que el diseño curricular está establecido para las escuelas completas y no para escuelas multigrado. 


\section{CONCLUSIONES}

En lo que respecta a describir la gestión pedagógica y académica de los docentes para el desarrollo de los contenidos curriculares del programa de inglés de la básica general, (primaria), en las escuelas multigrado, con la finalidad de lograr la equidad de la enseñanza. Se evidenció:

- Los docentes en su mayoría solo cuentan con un conocimiento básico para enseñar esta materia, es evidente que existe debilidad a la hora de su desarrollo, lo que se atribuye básicamente a las diversas responsabilidades que deben cumplir en la escuela multigrado.

- Se demuestra que la capacitación del docente en el ámbito de inglés es débil, su enseñanza carece de eficacia considerando que no se cumple con las horas establecidas ya que en las escuelas multigrado esta asignatura se desarrolla en función de sus necesidades. Por otro lado, no hay un documento que haga el ajuste correspondiente, ya que se les da prioridad a las asignaturas fundamentales y, además, los maestros señalaron que tienen un conocimiento básico del idioma inglés.

- Con relación a la propia gestión pedagógica de los docentes, se puede señalar que al carecer del dominio del idioma inglés y tener establecido un horario determinado para desarrollar el pensum curricular del grado correspondiente, en la escuela multigrado el inglés se desarrolla cuando las condiciones así lo permiten, lo que se convierte en una barrera, ya que tampoco cuentan con una biblioteca ni con internet para hacer uso de diferentes software y programas que apoyarían la gestión pedagógica.

- En cuanto a la efectividad de la gestión curricular y los elementos obstaculizadores de la propia gestión, se puede concluir que todo lo actuado y evidenciado en la unidad de análisis conformado por tres centros educativos, nos permite señalar que no existe efectividad para desarrollar la gestión que se espera, con la finalidad de lograr que los estudiantes de los centros multigrado tengan las mismas oportunidades que tienen los estudiantes de las escuelas urbanas.

Finalmente, luego de realizado el estudio y buscar el sustento teórico de las oportunidades que tienen los estudiantes de las escuelas multigrado y hablar de equidad en la formación que se les 
brinda, podemos señalar que evidentemente no se mira la equidad como la igualdad que en la formación deben tener todos los estudiantes del sistema sin importar el tipo de centro educativo en el que reciben esta formación: Ya que el propio Ministerio de Educación ve la equidad solo como cobertura y no desde el aspecto pedagógico del conocimiento que deben tener como derecho propio cada uno de los estudiantes que se ubican de frontera a frontera en nuestro país.

\section{REFERENCIAS}

Alcalá, C. (2013). La enseñanza del inglés en los centros rurales. Ediciones UMC.

Bracho González T. \& Hernández Fernández J. (2009). Equidad Educativa: Avances en la definición de su concepto. X Congreso nacional de investigación Educativa | área 10: interrelaciones educación-sociedad.

Bonhomme, M., Cox, C., Tham, M., y Lira, R. (2015). Aprendizaje de la Ciudadanía, Contextos, Experiencias y Resultados. Centro de estudios de políticas y prácticas en educación.

Carmena, D. y Regidor, R. (2014). Oportunidades de aprendizaje y rendimiento en matemáticas. PREALC.

Cameron, L. (2001). Teaching Languages to Young Learners. Cambridge University Press 1920.

Casarini, S. (2010). Enseñanza multigrado. Una visión general. Ediciones Internacional de Desarrollo Educativo.

Emma, F. (2016). ¿Aprenden igual los alumnos de una escuela urbana y de una escuela rural?. Ediciones McGraw-Hill. 
Guillén, F. (2016). El aprendizaje de lenguas extranjeras en Educación Infantil en la Comunidad de Madrid: perfil, percepciones y metodologías de los docentes. Revista Didáctica. Lengua y Literatura 111 2016, vol. 28, 87-111 91. Downloads/54090Texto\%20del\%20artículo-103578-2-10-20161124.pdf

Jiménez, D. (2016). La escuela rural. Trillas.

LEY No. 2 (14 de enero de 2003). Publicada en la Gaceta Oficial No. 24720 de 16 de enero de 2003, https://docs.panama.justia.com/federales/leyes/2-de-2003-jan-16-2003.pdf

Ministerio de Educación. (2018). Informe estadístico del sistema educativo.

https://www.meduca.gob.pa/direccion-plane/estadisticas

Ministerio de Educación. (2019). Plan estratégico de Educación. 2019-2024 https://www.meduca.gob.pa/sites/default/files/Plan\%20Estrate\%CC\%81gico\%20de\%20 Educaci\%CC\%81n\%20MEDUCA\%202019-UV\%20editado\%20(1).pdf. 